\title{
OBITUARIO
}

\section{In Memoriam doctor Juan Leónidas Tarazona Barboza (1952-2015)}

\section{In Memoriam doctor Juan Leónidas Tarazona Barboza (1952-2015)}

\section{Leonardo Romero}

Facultad de Ciencias Biológicas, Universidad Nacional Mayor de San Marcos. Apartado Postal 11-0058, Lima 11, Perú. Email Leonardo Romero: Iromeroc@unmsm.edu.pe

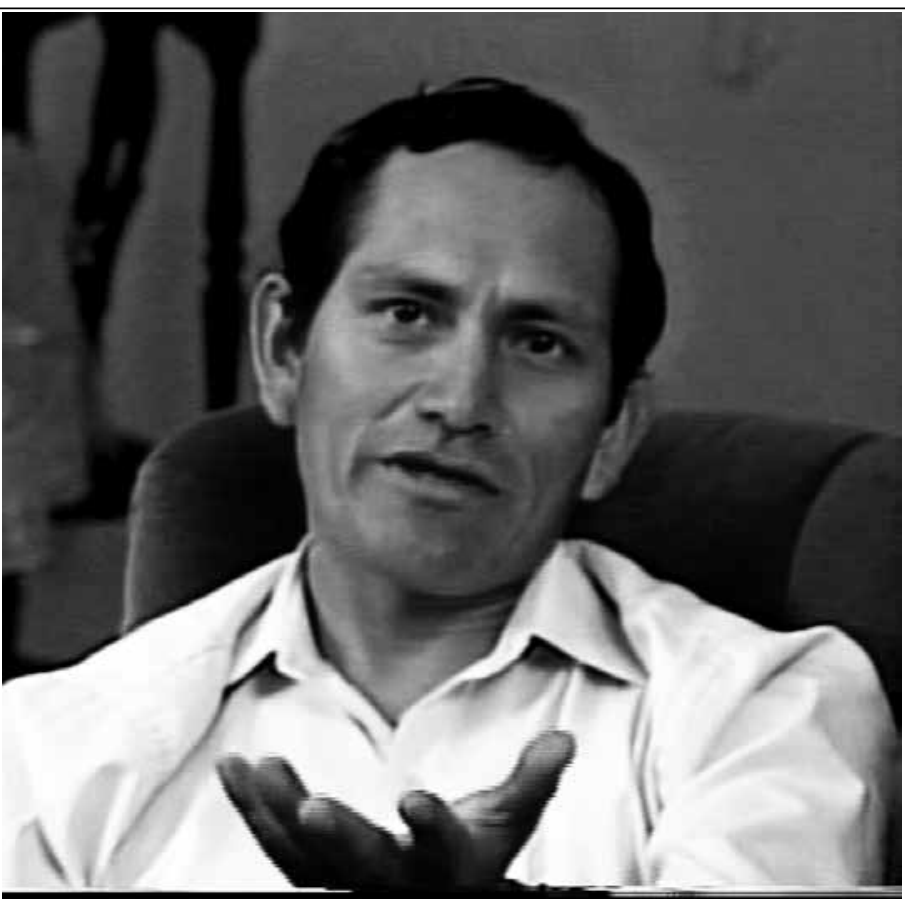

Doctor Juan Leónidas Tarazona Barboza.

"Basicamente, me considero un buscador de la verdad"

Juan Tarazona, nació el 28 de enero de 1952 y tuve el honor de ser espectador de una parte de su trayectoria académica, desde que fui su alumno, su ayudante, y después ya como colega. El 14 de mayo de 2015 falleció el doctor Juan Leónidas Tarazona Barboza, un alejamiento inesperado, porque la imagen que siempre prodigo el profesor Tarazona fue la de un joven incansable, ávido de emprender investigaciones y afrontar los retos académicos de ligar la ciencia a la sociedad. Siempre esperare verlo así. En estas lineas, como espectador siento que solo podre describir una parte de su trayectoria académica, solo las facetas que pude apreciar, solo el corto momento en que pude aprender de él y colaborar en sus investigaciones.

Citación:

Romero L. 2015. In Memoriam doctor Juan Leónidas Tarazona Barboza

(1952-2015). Revista peruana de biología 22(2): 139 - 144 (Agosto 2015).

doi: http://dx.doi.org/10.15381/rpb.v22i2.11350 
Juan Tarazona inició oficialmente su labor docente en 1971 en la Universidad Nacional Mayor de San Marcos, en lo que hoy es la Facultad de Ciencias Biológicas. Ya en esos años participaba en estudios de la diversidad del intermareal rocoso de Lima. Carlos Paredes, quien se encontraba realizando su trabajo de tesis doctoral, lo había aceptado como asistente. La tesis de Carlos Paredes se convirtió posteriormente en el registro taxonómico más completo de invertebrados de la orilla rocosa y en la descripción biocenotica más utilizada en el Perú hasta la actualidad $^{1}$ para describir ese ambiente. Podemos inferir que estas labores lo orientaron y prepararon para elegir su primer tema de investigación, los poliquetos. Otros temas que empezó a abordar en esa época fueron la ecología de playas arenosas y de los fondos blandos. Sin embargo, debemos tomar en cuenta que en la década de los años 70 el Perú no era un escenario propicio para el desarrollo académico, es así que el empeńo de Tarazona por realizar investigaciones lo marcaba ya como un individuo excepcional.

En el año 1974, Tarazona sustento su tesis de bachiller "Poliquetos errantes de la zona litoral del departamento de Lima". En ese año también da inicio la publicación de la Revista Peruana de Biología, y podemos observar uno de sus trabajos en el segundo número del primer volumen; sin embargo su primera publicación, aunque fue sobre poliquetos se refirió a otro grupo diferente del de su tesis (poliquetos errantes), al grupo conocido por ese entonces como los poliquetos sedentarios. Mientras tanto, él como docente participaba en cursos como Hidrobiología y Ecología Marina, sobre los cuales empezó a trabajar para estructurarlos. También, organizaba cursos de extensión con investigadores extranjeros que visitaban el país, y participaba en cruceros internacionales en estudios de las condiciones hipóxicas frente al Perú.

Juan Tarazona era un profesor e investigador excepcional. A fines de los ańos $70 \mathrm{y}$ principios de los 80 , la universidad peruana pasaba por una seria crisis académica, por ejemplo no habían libros ni subscripciones a revistas. Él se subscribía al Current Contents y de esa manera nos enterábamos de las publicaciones más recientes y solicitábamos las más interesantes. Esta práctica le aseguraba a él y al grupo que le rodeaba las condiciones para lograr una autoformación académica, y estar al día con las nuevas propuestas de investigación; aspecto que para Tarazona era trascendente y de alta responsabilidad.

A principios de la década de los 80 , Juan Tarazona ya había empezado sus muestreos en la bahía de Ancón y conformado un grupo de estudios (DePSEA, grupo para el Desarrollo Pesquero en áreas Someras del Ecosistema de Afloramiento). Este grupo formado por docentes y estudiantes de la facultad jugaron un papel importante en los siguientes años. El grupo DePSEA fue creado motivado por la presentación de una propuesta de proyecto para conformar una cartera que debería ser apoyado por el Programa de Investigación Colaborativa para el Apoyo de la Evaluación de los Stock de pesca artesanal (Stock Assessment CRSP) que sería financiado por la US-AID. El proyecto que propuso Tarazona se llamó "Programa piloto para el desarrollo pesquero en áreas someras del afloramiento peruano", y englobaba sus concepciones de investigación en ese momento. También, a principios de los ańos 80, empezaba a trabajar el Programa Cooperativo Peruano Alemán (PROCOPA), y su primer director Wolf Arntz se convertiría en un muy importante colaborador para los estudios del bentos que Tarazona ya había empezado a realizar, es decir estudios enfocados en áreas costeras someras, su dinámica, sus recursos, sus condiciones ambientales como las hipoxias y los impactos de los eventos El Niño.

Aunque el evento El Niño 72-73 había causado una crisis de la anchoveta y los científicos comenzaban a tomar en cuenta el impacto de esas perturbaciones ambientales, El Niño 82-83 fue crucial para que la comunidad científica le diera a este evento la importancia que se merecía, en particular para Juan Tarazona

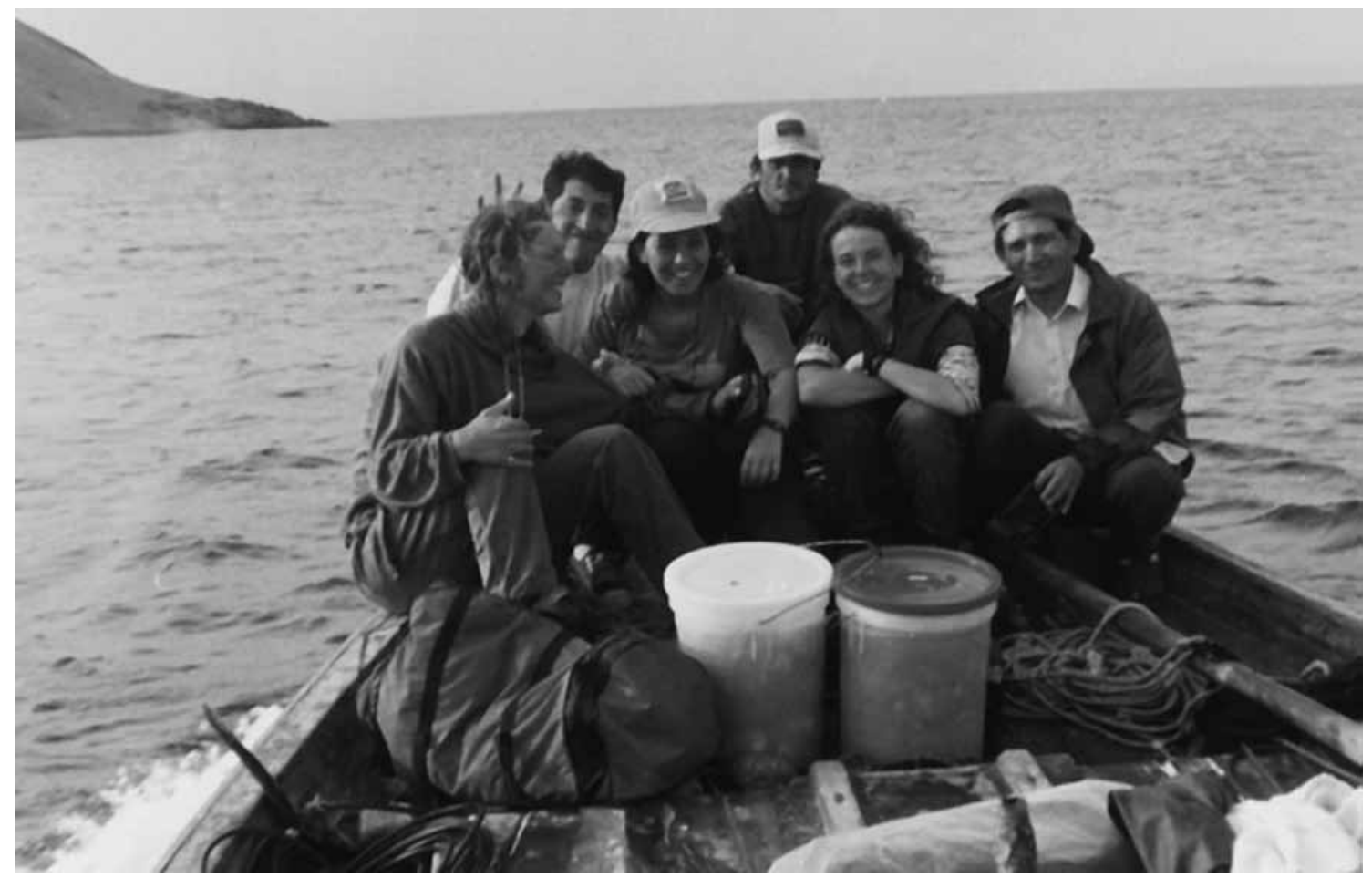

Doctor Juan Tarazona (derecha) en trabajo de campo en Bahia Independencia (RIBEN) 


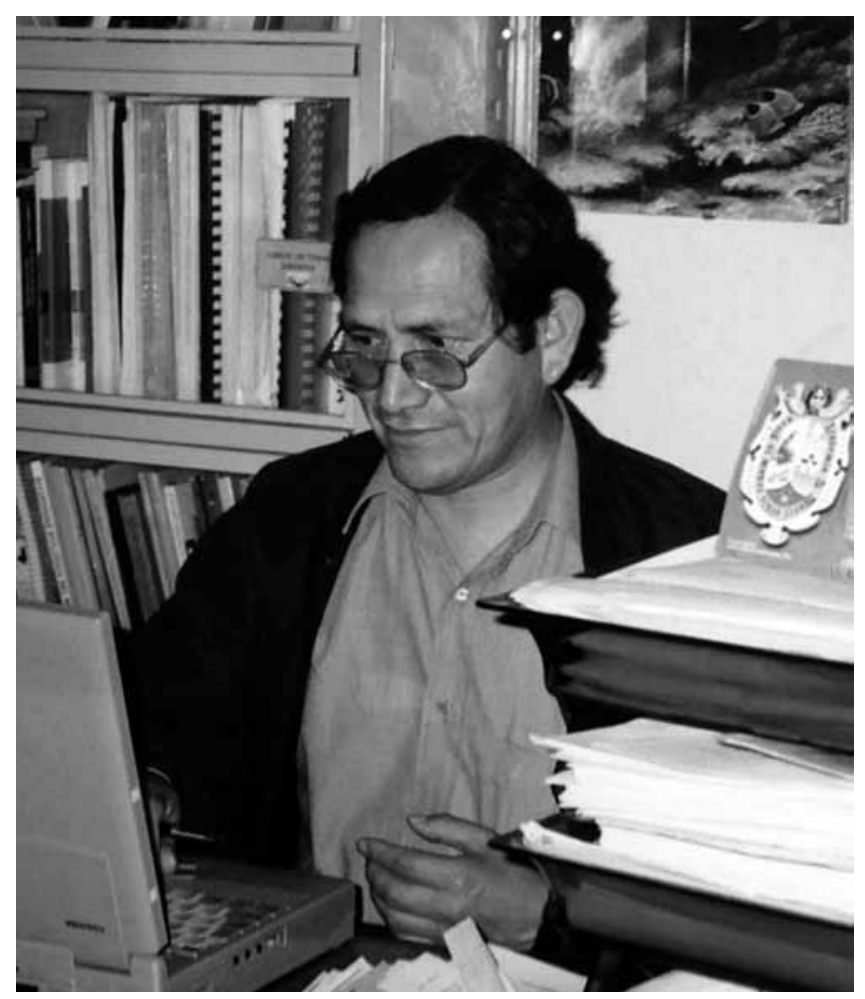

Doctor Juan Tarazona en su oficina - laboratorio (Foto cortesia de Tania Peña).

fue un evento decisivo. La ocurrencia del evento El Niño 82-83 fue el escenario que le permitió a Tarazona desplegar todo su potencial de investigador y científico, él había acumulado hasta ese momento bastante información sobre la ecología del bentos marino en diversos hábitats, intermareales y de profundidad, de fondos duros y blandos, de macrobentos y meiobentos; esto fue lo que le permitió describir e interpretar lo que en ese momento sucedía con el bentos marino. Posteriormente, sus observaciones plasmadas en publicaciones se convirtieron en referente de nuestros actuales conocimientos sobre el impacto de El Niño en el bentos.

Unos años más tarde, con apoyo del US-AID (Agencia de los Estados Unidos para el Desarrollo) se iniciaron los estudios comparativos entre las bahías de Ancón e Independencia (Pisco), las que le permitieron ampliar el estudio de diferentes aspectos de la problemática de la hipoxia de los bentos someros en el Perú. El proyecto "Mecanismos y procesos que controlan la colonización y recuperación post-catastrófica de recursos bentónicos de importancia económica en dos áreas de diferente productividad del sistema de afloramiento peruano" se llevó a cabo entre 1986 y 1988, permitiendo realizar experimentos y observaciones que describirían la ecología del bentos, en particular los procesos de colonización.

Mientras tanto, los resultados y observaciones sobre el evento El Nińo 1982-83, ya comenzaban a ser citados por otros trabajos y Juan Tarazona a principios de 1988 viajaba a emprender su doctorado. La calidad de los trabajos de Juan Tarazona se vislumbraron claramente cuando en la importante revisión de Peter Glynn sobre los impactos de El Niño 1982-83, se mencionó:

"A well-documented study by Tarazona el al. (252) showed profound changes in benthic community composition, involv-

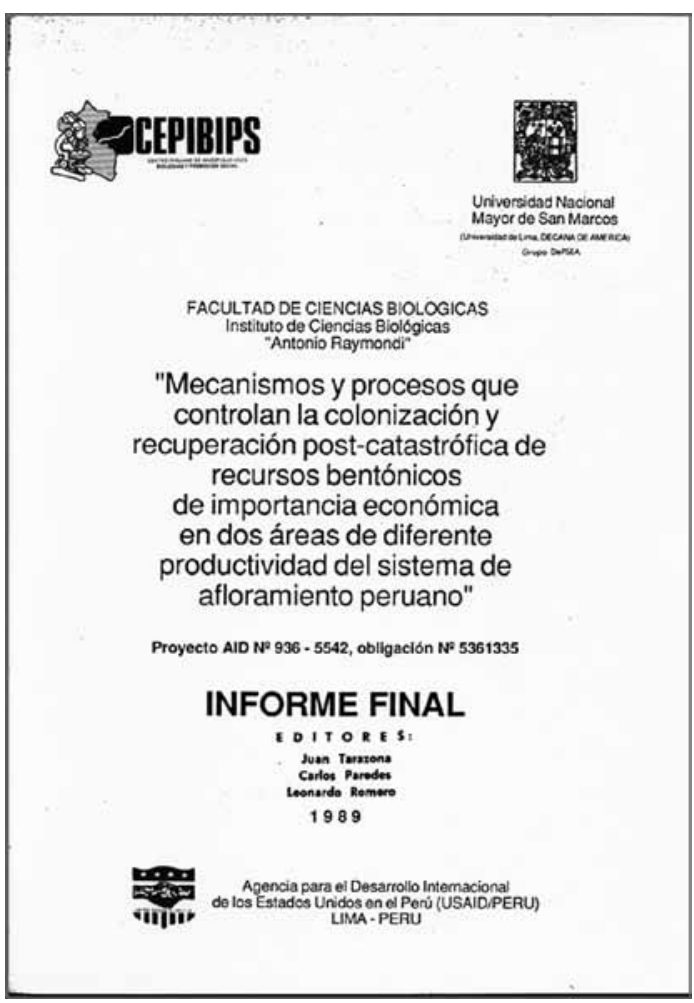

ing the recruitment of formerly rare and seasonal species (algae, a brachiopod, and polychaete worms) and tropical species (polychaete worms, a cirriped, stomatopod, crabs, shrimp, a gastropod, and bivalves) to the dead mussel assemblages mentioned above. Many of the warm water invaders demonstrated a competitive advantage in space utilization and significant impacts as predators. These profound changes led Tarazona el

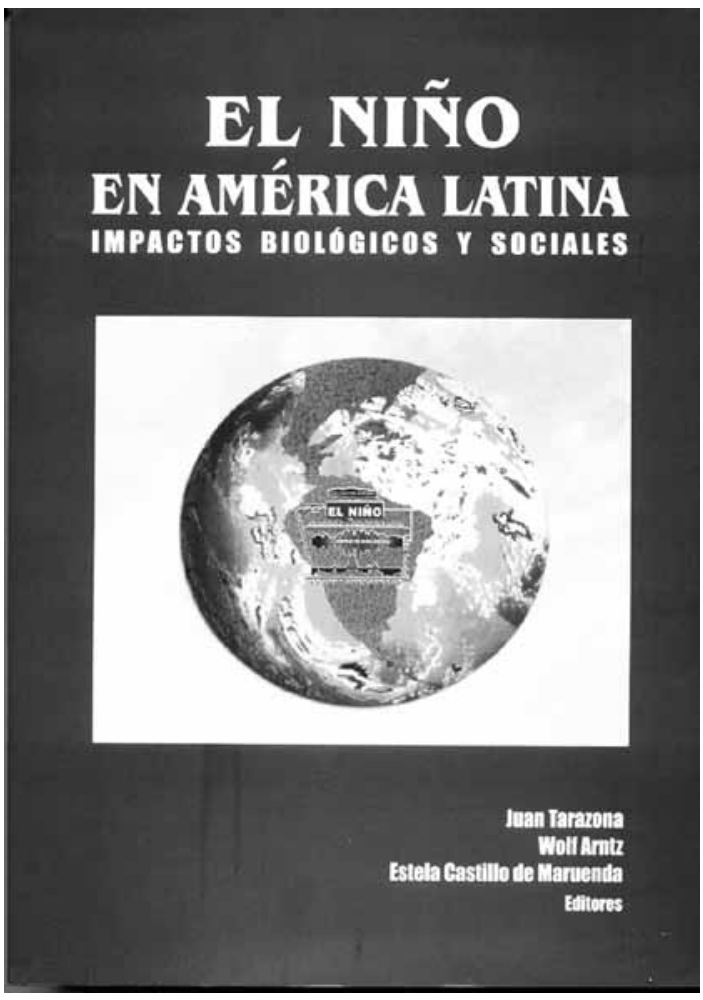


al. (252) to regard severe El Niño disturbances and subsequent recruitment events as influential controls of nearshore, benthic community structure" ${ }^{2}$.

En el año 1990 sustentó su grado de Doktors Der Naturwissenschaften (Dr. rer. nat.) obtenido en la Die Universität Bremen verleiht durch den, Alemania, con la tesis Disturbance and stress associated to El Niño and their significancce for the macrobenthos of shallow areas of the Peruvian upwelling ecosystem. Sus temas de investigación y la calidad de los mismos le valieron el reconocimiento de la comunidad científica nacional e internacional. Cuando él regresó a Perú, su condición de investigador de alto nivel, lo llevó a vincularse con el CONCYTEC oficialmente desde el 2002, donde se desempeñó en varios cargos, como el de Asesor, Director General de Programas, Director de Ciencia y Tecnología (2006 - 2008) y últimamente el de Director del Fondo Nacional de Ciencia, Tecnología e Innovación Tecnológica (FONDECYT) (2011 - 2012). Durante este periodo, él fue promotor de investigaciones sobre Biodiversidad y Cambio Climático Global, temas que impulso con varias publicaciones y ediciones de libros, siempre claro en sus ideas y de cómo deberían de enfocarse los problemas, subrayando siempre lo rápido en que sucedían los cambios a nivel mundial y la actitud que debemos tener hacia ellos, por ejemplo cuando nos dice en un trabajo que fue desarrollado a fines de los años 90, pero publicado en el 2009: "Finalmente, hay que destacar que la mayor preocupación sobre el impacto del cambio climático global se ha centrado en las consecuencias de largo plazo, pero nosotros debemos desplegar también los máximos esfuerzos por resolver los efectos transicionales que podrían ser muy severos en las décadas más cercanas" 3 .

El doctor Juan Tarazona fue líder en varios importantes proyectos como la RIBEN (Red sobre Impactos Biológicos de los Eventos El Niño), el CENSOR (Climate variability and El Niño Southern Oscillation) y el proyecto "Impacto de la variabilidad y cambio climático en el ecosistema de manglares de Tumbes". También participó en la "Red de Investigación Científica en Cambio Climático de la Alianza del Pacífico" y la formulación del proyecto de "Cooperación Científica en Materia de Cambio Climático: Monitoreo de la biodiversidad de nueva generación para apoyar procesos de adaptación y mitigación al cambio climático”. Además, participó como colaborador y parte del equipo que elaboró la publicación "Oportunidades de colaboración en Investigación sobre cambio climático en los países de la Alianza del Pacífico". Fue presidente del Consejo Consultivo de la Facultad de Biología Marina y Econegocios de la Universidad Científica del Sur y miembro del Consejo Directivo del Instituto Geofísico del Perú.

El doctor Juan Tarazona fue Académico Titular de la Academia Nacional de Ciencia y Tecnología (ANCyT) y Compartió el Premio Nobel de la Paz 2007 otorgado a los autores del IV Informe de Evaluación del Panel Intergubernamental de Cambio Climático.

Juan Tarazona fue el profesor que estructuró, diseñó y dictó los cursos de Hidrobiología y Ecología Marina por muchos años, cursos que con sus clases, muestreos de campo y viajes de investigación orientaron a varias generaciones de biólogos. Fue asesor de muchas tesis, un maestro dinámico, con muchas actividades a la vez, y con muchas ideas para desarrollar. Recordar a Juan Tarazona es traer a la memoria al profesor exigente, que nos decia que "no hay que acostumbrarse a las cosas malas". Al investigador perseverante, que nos demostraba con sus acciones todo lo que podíamos hacer y tenemos que hacer por la responsabilidad que la ciencia nos exige. Juan Tarazona fue un investigador que podía definir bien la situación de todo buen científico peruano; él lo había vivido en su afán de realizar investigación y nos decía: "Para un científico, en un país como el nuestro, lo más difícil es hacer ciencia de alto nivel, y poder mantenerse en una línea de investigación, en una especialización y tener la holgura económica para vivir decentemente"; esa "ciencia de alto nivel" es "hacer las cosas bien", la responsabilidad que tenemos y que el doctor Tarazona nos lo señaló de múltiples maneras.

Estoy seguro que todo esto que pude ver como espectador de la vida de Juan Tarazona fueron solamente unas facetas de su vida, porque él se consideraba sobre todo un buscador de la verdad, no solo en la ciencia, sino también en la vida cotidiana y en las multiples formas en que la verdad pudiera encontrarse. Por último, otra señal de la existencia de esas otras facetas se dejan entrever en una entrevista a raíz de los eventos El Niño y la RIBEN, cuando él ante la pregunta ¿Qué hubiera sido usted si no hubiese sido biólogo? respondió "Si no hubiese sido biólogo [...] hubiera sido un filósofo".

\section{Publicaciones científicas del doctor Juan Tarazona}

1. J. Tarazona, «Poliquetos errantes de la zona litoral del Departamento de Lima», Tesis de Bachiller, Universidad Nacional Mayor de San Marcos, Lima, Perú, 1974.

2. J. Tarazona, «Lista de poliquetos sedentarios hallados en el Perú», Rev peru biol, vol. 1, n. ${ }^{\circ}$ 2, pp. 164 - 167, 1974.

3. J. Tarazona, V. Ishiyama, y S. Barrera, «Ovogénesis en el pejerrey, Odontesthes regia regia (H.)», Revista de Ciencias, UNMSM, vol. 71, n. ${ }^{\circ} 1$, pp. $29-37,1979$.

4. C. Paredes y J. Tarazona, «Las comunidades de mitilidos del mediolitoral rocoso del departamento de Lima", Rev peru biol, vol. 2, n. ${ }^{\circ}$ 1, pp. 59 - 72, 1980.

5. J. Tarazona, «Ecología de los bancos de pelecípodos en las playas arenosas del Perú y pautas para su conservación», en Anales del Primer Panel sobre los Moluscos del Perú (agosto 1981), 1981, p. 52-61.

6. R. Rosenberg, W. E. Arntz, E. C. de Flores, L. A. Flores, G. Carbajal, I. Finger, y J. Tarazona, «Benthos biomass and oxygen deficiency in the upwelling system off Peru", Journal of Marine Research, vol. 41, n. ${ }^{\circ}$ 2, pp. 263-279, may 1983.

7. J. Tarazona, "Modificaciones de la infauna bentonica de una bahía con deficiencia de oxigeno durante el nino 1982 - 83", Revista de la Comisión Permanente del Pacifico Sur, vol. 15, pp. 223-238, 1984.

8. W. Arntz, A. Landa, y J. Tarazona, Eds., El Niño. Su impacto en la fauna marina, vol. Volumen extraordinario. Callao: Instituto del Mar del Perú -IMARPE, 1985.

9. L. Hoyos, J. Tarazona, B. Shiga, y V. Chiong, «Algunos Cambios en la Ictiofauna y sus Relaciones Tróficas durante el Fenómeno El Niño en la Bahía de Ancón.», En: Arntz, W., A. Landa y J. Tarazona (eds.). El Niño: Su impacto en la Fauna Marina. Boletín - Instituto del Mar del Perú, vol. Volumen extraordinario, pp. 163-171, 1985.

10. J. Tarazona, C. Paredes, L. Romero, V. Blaskovic, S. Guzmán, y S. Sanchez, "Características de la vida planctónica y colonización de los organismos epilíticos durante el fenómeno "El Niño"», En: Arntz, W., A. Landa y. Tarazona (eds.). El Niño: Su impacto en la Fauna Marina. Boletín - Instituto del Mar del Perú, vol. Volumen extraordinario, pp. $41-50,1985$.

11. N. Rojas, J. Tarazona, y V. Ishiyama, "Ciclo de reproducción y escala de madurez gonadal en el "caracol" Thais (Stramonita) chocolata (Duclos, 1832)», Revista de Ciencias, UNMSM, vol. 74, n. ${ }^{\circ}$ 1, pp. 117 - 129, 1986.

12. J. Tarazona y W. Arntz, «Bacterias filamentosas gigantes en fondos someros de la Bahía de Ancón», Revista de Ciencias, UNMSM, vol. 74, n. ${ }^{\circ} 1$, pp. 178-180, 1986 . 
13. J. Tarazona, C. Paredes, y M. Igreda, «Estructura del macrobentos en las playas arenosas de la zona de Lima, Perú», Revista de Ciencias, UNMSM, vol. 74, n. ${ }^{\circ}$ 1, pp. 103 - 116, 1986.

14. L. J. Zegers, V. Ishiyama, y J. Tarazona, "Contribución al conocimiento biologico pesquero del recurso "chanque" Concholepas concholepas (Bruguiere, 1789) (Gastropoda, Muricidae) en la zona de Pisco, Perú.», Revista de Ciencias, UNMSM, vol. 74, n. ${ }^{\circ}$ 1, pp. $87-102,1986$.

15. C. Talledo, V. Ishiyama, y J. Tarazona, «Reproducción de Donax peruvianus. Deshayes 1985 en el área de caleta San José y playa Naylamp, Lambayeque», Biota, vol. 13, n. ${ }^{\circ}$ 93, pp. 4 - 16, 1987.

16. W. E. Arntz, T. Brey, J. Tarazona, y A. Robles, «Changes in the structure of a shallow sandy-beach community in Peru during an El Niño event», South African Journal of Marine Science, vol. 5, n. ${ }^{\circ}$ 1, pp. 645-658, jun. 1987.

17. W. Arntz y J. Tarazona, «Una retrospectiva a El Nińo 1982-83. ¿qué hemos aprendido?», En: Salzwedel H. \& Landa A. (Eds.). Recursos y dinámica del ecosistema del afloramiento peruano. Boletin Instituto del Mar Perú Callao, vol. Volumen extraordinario, pp. 353 - 364, 1988.

18. C. Paredes, J. Tarazona, E. Canahuire, L. Romero, y O. Cornejo, «Invertebrados Macro-Bentónicos del área de Pisco, Perú», En: Salzwedel H. \& Landa A. (Eds.). Recursos y dinámica del ecosistema del afloramiento peruano. Boletin Instituto del Mar Perú Callao, vol. Volumen extraordinario, pp. 121 - 132, 1988.

19. J. Tarazona, H. Salzwedel, y W. Arntz, «Oscillations of macrobenthos in shallow waters of the Peruvian central coast induced by El Nino 1982-83", Journal of Marine Research, vol. 46, n. ${ }^{\circ} 3$, pp. $593-611,1988$.

20. J. Tarazona, H. Salzwedel, y W. Arntz, "Positive effects of "El Niño" on macrozoobenthos inhabiting hypoxic areas of the Peruvian upwelling system», Oecologia, vol. 76, n. ${ }^{\circ}$ 2, pp. 184190, 1988.

21. J. Tarazona, W. Arntz, y L. Hoyos, «Repartición de los recursos alimenticios entre tres peces bentófagos frente al Perú antes, durante y después de El Niño 1982-83", En: Salzwedel H. \& Landa A. (Eds.). Recursos y dinámica del ecosistema del afloramiento peruano. Boletin Instituto del Mar Perú Callao, vol. Volumen extraordinario, pp. $107-114,1988$.

22. J. Tarazona, C. Paredes, L. Romero, y S. Guzman, «La recolonización de las comunidades de mitílidos en la costa central del Perú después de El Niño 1982-1983", En: Salzwedel H. \& Landa A. ( Eds. ). Recursos y dinámica del ecosistema del afloramiento peruano. Boletin Instituto del Mar Perú Callao, vol. Volumen extraordinario, pp. $115-120,1988$

23. M. Tokeshi, C. Estrella, y J. Tarazona, «Estudio preliminar de las relaciones predador-presa en (Heliaster helianthus Asteroidea: Echinodermata)", En: Salzwedel H. \& Landa A. (Eds. ). Recursos y dinámica del ecosistema del afloramiento peruano. Boletin Instituto del Mar Perú Callao, vol. Volumen extraordinario, pp. $141-145,1988$.

24. J. Tarazona, C. Paredes, y L. Romero, Eds., Mecanismos y procesos que controlan la colonización y recuperación post-catastrófica de recursos bentónicos de importancia económica en dos áreas de diferente productividad del sistema de afloramiento peruano, Informe Final. Proyecto AID No 936-5542, obligación No 5361335. Lima, Perú, 1989.

25. M. Tokeshi, L. Romero, y J. Tarazona, «Spatial coexistence of mussel-associated, free-ranging polychaetes in a subtropical intertidal habitat», Journal of Animal Ecology, vol. 58, n. ${ }^{\circ}$ 2, pp. 681-692, 1989.

26. W. E. Arntz y J. Tarazona, «Effects of El Niño 1982-1983 on benthos, fish and fisheries off the South American Pacific Coast", en Effects of El Niño 1982-83 on Benthos, Fish and Fisheries off the South American Pacific Coast, vol. 52, P. W. Glynn, Ed. 1990, pp. $323-360$.

27. W. E. Arntz y J. Tarazona, «El Niño 1982-83 im Rückblick - was haben wir gelernt?», Biologie in Unserer Zeit - BIOL UNSERER ZEIT, vol. 20, n. ${ }^{\circ}$ 6, pp. 289-296, 1990.

28. J. Tarazona, «Disturbance and stress associated to El Niño and their significance for the macrobentos of shallow areas of the peruvian upwelling ecosystem», Ph.D. thesis, University of Bremen, Bremen, Germany, 1990.
29. J. Tarazona, E. Canahuire, H. Salzwedel, T. Jeri, W. Arntz, y L. Cid, «Macrozoobenthos in two shallow areas of the Peruvian upwelling ecosystem", en Estuaries and coasts: spatial and temporal intercomparisons, vol. ECSA19 Symposium, University of Caen, France, M. Elliott y J.-P. Ducrotoy, Eds. Amsterdam: Olsen \& Olsen, 1991, pp. 251-258.

30. W. E. Arntz, J. Tarazona, V. A. Gallardo, L. A. Flores, y H. Salzwedel, «Benthos communities in oxygen deficient shelf and upper slope areas of the Peruvian and Chilean Pacific coast, and changes caused by El Niño», Geological Society, London, Special Publications, vol. 58, n. ${ }^{\circ}$ 1, pp. 131-154, ene. 1991.

31. J. Tarazona y C. Paredes, «Impacto de los eventos El Niño sobre las comunidad bentónicas de de playa arenosa durante 19761986", en Paleo ENSO Records, extended abstract, L. Ortlieb y J. Marchare, Eds. Lima, Perú: ORSTOM-CONCYTEC, 1992, pp. 299-333.

32. J. Tarazona, «Biodiversidad en el Mar Peruano», Actas, Academia Nacional de Ciencia y Tecnología, vol. Tomo 2, n. ${ }^{\circ} 3$, pp. 9-12, 1994.

33. J. Tarazona, W. E. Arntz, y E. Canahuire, «Impact of two "El Niño" events of different intensity on the hypoxic soft bottom macrobenthos off the central peruvian coast", Marine Ecology, vol. 17, n. ${ }^{\circ} 1-3$, pp. 425-446, 1996.

34. H.-J. Urban y J. Tarazona, «Effects of El Nino/southern oscillation on the population dynamics of a Gari solida population (Bivalvia: Psammobiidae) from Bahia Independencia, Peru», Marine Biology, vol. 125, n. ${ }^{\circ}$ 4, pp. 725-734, 1996.

35. F. Cardoso, J. Tarazona, y C. Paredes, «Aspectos biológicos del calamar patagónico Loligo gahi (Cephalopodo: Loliginidae) en Huarmey, Perú», Rev peru biol, vol. 5, n. ${ }^{\circ}$ 1, pp. 009 -014, 1998.

36. C. Paredes, J. Tarazona, E. Canahuire, L. Romero, O. Cornejo, y F. Cardoso, «Presencia de moluscos tropicales de la provincia panameña en la costa central del Perú y su relación con los eventos "El Niño"”, Rev peru biol, vol. 5, n. . 2, pp. 123 - 128, 1998.

37. S. Sanchez, J. Tarazona, R. Flores, M. Maldonado, y G. Carbajal, "Características del fitoplancton de invierno en Bahía Independencia, Perú», En: Salzwedel H. \& Landa A. (Eds. ). Recursos y dinámica del ecosistema del afloramiento peruano. Boletin Instituto del Mar Perú Callao, vol. Volumen extraordinario, pp. $57-66,1998$.

38. J. Tarazona, P. Aguilar, O. Tovar, H. Ortega, y G. Lamas, «Estado del conocimiento de la diversidad biológica en el Perú: una sinopsis», en La Diversidad Biológica de Iberoamérica Vol. III, vol. Volumen especial, G. Halffter, Ed. Xalapa, México: CYTED, Instituto de Ecología, 1998.

39. J. Tarazona, W. E. Arntz, E. Canahuire, Z. Ayala, y A. Robles, «Modificaciones producidas durante "El Niño" en la infauna bentónica de areas someras del ecosisitema de afloramiento peruano", In: Arntz, W., Landa, A. and Tarazona, J. (eds.), «El Niño»: Su impacto en la fauna marina. Boletín - Instituto del Mar del Perú, vol. Volumen extraordinario, pp. 55-63, 1998.

40. J. Tarazona y S. Valle, "La diversidad biológica en el mar peruano", en La Diversidad Biológica de Iberoamérica Vol. III, vol. Volumen especial, G. Halffter, Ed. Xalapa, México: CYTED, Instituto de Ecología, 1998.

41. E. Fernández, C. Córdova, y J. Tarazona, «Condiciones de la pradera submareal de Lessonia trabeculata en la isla Independencia durante "El Nińo 1997-98"», Rev peru biol, vol. 6, n. ${ }^{\circ} 3$, pp. 047 - 059, 1999

42. C. Paredes, F. Cardoso, y J. Tarazona, «Invertebrados del intermareal rocoso del departamento de Lima, Perú: Una lista comentada de especies", Rev peru biol, vol. 6, n. ${ }^{\circ} 2$, pp. $143-151,1999$.

43. E. Ramos, A. Indacochea, y J. Tarazona, «Impacto de "El Nińo 1997-98" sobre el asentamiento larval de algunos invertebrados marinos bentónicos de bahía Independencia, Pisco -Perú», Rev peru biol, vol. 6, n. ${ }^{\circ} 3$, pp. 060 - 068, 1999.

44. J. Tarazona y $S$. Valle, «Impactos potenciales del cambio climático global sobre el ecosistema marino peruano", en Perú: Vulnerabilidad frente al cambio climático: Aproximaciones a la experiencia con el fenómeno El Niño, Primera edición., B. Marticorena, Ed. Lima: Consejo Nacional del Ambiente -CONAM, 1999, pp. 95 - 111.

45. J. Tarazona B., J. T. B, A. Indacochea, S. Valle, C. Córdova, N. Ochoa, W. Serrano, y T. Peńa, "Impacto de "El Niño 1997-98" sobre el ecosistema marino somero de la costa central del Perú», Rev peru biol, vol. 6, n. ${ }^{\circ}$ 3, pp. 018 - 031, 1999. 
46. J. Tarazona y W. Arntz, «The Peruvian Coastal Upwelling System", en Coastal Marine Ecosystems of Latin America, D. U. Seeliger y D. B. Kjerfve, Eds. Springer Berlin Heidelberg, 2001, pp. 229-244.

47. J. Tarazona, W. Arntz, y E. Castillo De Maruenda, Eds., El Niño en América Latina: Impactos biológicos y sociales. Lima: Consejo Nacional de Ciencia y Tecnologia- CONCYTEC, 2001.

48. J. Tarazona, W. Arntz, S. Valle, y T. Peña, «Los indices de El Niño y del impácto sobre las comunidades bentónicas», en $E l$ Niño en América Latina: Impactos biológicos y sociales, J. Tarazona, W. Arntz, y E. Castillo De Maruenda, Eds. Consejo Nacional de Ciencia y Tecnologia- CONCYTEC, 2001, pp. 113-126.

49. J. Tarazona y W. E. Arntz, "Impacts of El Niño Events on the Coastal Benthos of the Eastern Pacific Upwelling Systems», Investigaciones Marinas, vol. 30, n. ${ }^{\circ}$ 1, 2002.

50. S. Valle, J. Tarazona, A. Indacochea, E. Ramos, y W. Serrano, «Variabilidad inducida por el ciclo del ENOS en la Densidad Poblacional de algunos invertebrados bentónicos de bahía Independencia, Pisco-Perú», en Bases Ecológicas y Socioeconómicas para el Manejo de los Recursos Vivos de la Reserva Nacional de Paracas, J. Mendo y M. Wolff, Eds. Universidad Nacional Agraria La Molina, 2002, pp. 68-76.

51. N. Ochoa y J. Tarazona, «Variabilidad temporal de pequeña escala en el fitoplancton de bahía independencia, Pisco, Perú», Rev peru biol, vol. 10, n. ${ }^{\circ} 1$, pp. 59-66, 2003.

52. J. Tarazona, D. Gutiérrez, C. Paredes, y A. Indacochea, «Overview and challenges of Marine Biodiversity Research in Peru", Gayana (Concepción), ene. 2003.

53. C. Paredes, F. Cardoso, y J. Tarazona, «Distribución temporal de moluscos y crustáceos tropicales en la Provincia Peruana y su relación con los eventos El Niño", Rev peru biol, vol. 11, n. ${ }^{\circ}$, pp. 213-218, 2004.

54. T. S. Peña, K. Johst, V. Grimm, W. Arntz, y J. Tarazona, «Population dynamics of a polychaete during three El Niño events: Disentangling biotic and abiotic factors", Oikos, vol. 111, n. $^{\circ} 2$, pp. 253-258, 2005.

55. W. E. Arntz, V. A. Gallardo, D. Gutiérrez, E. Isla, L. A. Levin, J. Mendo, C. Neira, G. T. Rowe, J. Tarazona, y M. Wolff, «El Niño and similar perturbation effects on the benthos of the Humboldt, California, and Benguela Current upwelling ecosystems», Advances in Geosciences, vol. 6, pp. 243-265, 2006.

56. V. Montecino, P. T. Strub, F. Chavez, A. Thomas, J. Tarazona, y T. Baumgartner, «Bio-physical interactions off western SouthAmerica», en The Global Coastal Ocean: Interdisciplinary Regional Studies and Syntheses, vol. 14A, A. R. Robinson y K. H. Brink, Eds. Cambrige, M.A.: Harvard University Press, 2006, pp. 329 - 390.

57. T. S. Peña, K. Johst, V. Grimm, W. E. Arntz, y J. Tarazona, "Disentangling the effects of El Niño on a population of the polychaete Sigambra bassi in the Bay of Ancón, Peru", Advances in Geosciences, vol. 6, pp. 161-166, 2006.

58. A. Fischlin, G. F. Midgley, J. Price, R. Leemans, B. Gopal, C. Turley, M. Rounsevell, P. Dube, J. Tarazona, y A. Velichko, "Ecosystems, their properties, goods, and services», en Climate change 2007: impacts, adaptation and vulnerability. Contribution of Working Group II to the Fourth Assessment Report of the Intergovernmental Panel on Climate Change, M. L. Parry, O. F. Canziani, J. P. Palutikof, P. J. Van der Linden, y C. E. Hanson, Eds. Cambridge, UK: Cambridge University Press, 2007, pp. 211-272.
59. J. Tarazona, R. Espinoza, M. Solís, y W. Arntz, «Crecimiento y producción somática de la concha de abanico (Argopecten purpuratus) en Bahía Independencia, Pisco (Perú) comparados entre eventos El Niño y La Niña", Revista de biología marina y oceanografia, vol. 42, n. ${ }^{\circ}$ 3, pp. 275-285, dic. 2007.

60. F. J. Hidalgo, F. N. Firstater, E. Fanjul, M. C. Bazterrica, B. J. Lomovasky, J. Tarazona, y O. O. Iribarne, "Grazing effects of the periwinkle Echinolittorina peruviana at a central Peruvian high rocky intertidal», Helooland Marine Research, vol. 62, n. ${ }^{\circ} 2$ SUPPL.1, pp. 73-83, 2008.

61. G. Sotil, J. Tarazona, R. Alvis, J. C. Francia, y B. Shiga, «Comparative evaluation of the DNA damage response in two Peruvian marine bivalves exposed to changes in temperature», Helgoland Marine Research, vol. 62, n. ${ }^{\circ}$ SUPPL.1, pp. 101-105, 2008.

62. J. Tarazona y E. Vilela, «Vulnerabilidad actual y futura al cambio climático en la bahía de Sechura y propuestas de adaptación en Perú», en Vulnerabilidad y adaptación al cambio climático en el Perú: Prioridades de ciencia y tecnología, Consejo Nacional de Ciencia, Tecnología e Innovación Tecnológica (CONCYTEC), Ed. Lima, Perú, 2009, pp. 53 - 66.

63. R. Espinoza, J. Tarazona, y J. Laudien, «Características de una población sobreexplotada de concha navaja, Ensis macha, en Bahía Independencia, Perú, durante el 2004", Revista Peruana de Biología, vol. 17, n. 3 3, pp. 285-292, 2010.

64. F. N. Firstater, F. J. Hidalgo, B. J. Lomovasky, J. Tarazona, G. Flores, y O. O. Iribarne, "Coastal upwelling may overwhelm the effect of sewage discharges in rocky intertidal communities of the Peruvian coast", Marine and Freshwater Research, vol. 61, n. ${ }^{\circ}$ 3, pp. 309-319, 2010.

65. B. J. Lomovasky, P. A. Gamero, L. Romero, F. N. Firstater, A. Gamarra Salazar, F. Hidalgo, J. Tarazona, y O. O. Iribarne, «The role of Argopecten purpuratus shells structuring the soft bottom community in shallow waters of southern Peru", Journal of Sea Research, vol. 106, pp. 14-26, dic. 2015.

\section{Literatura citada}

1 Carlos Paredes. "Contribución al conocimiento de los invertebrados del litoral rocoso del Departamento de Lima con estudio especial de la biocenosis de mitílidos», Tesis doctoral, Universidad Nacional Mayor San Marcos, Lima, Perú, 141 p. 1974.

2 Peter W. Glynn, «El Nino-Southern Oscillation 1982-1983: Nearshore Population, Community, and Ecosystem Responses», Annual Review of Ecology and Systematics, vol. 19, n. ${ }^{\circ}$ 1, pp. 309-346, 1988. [p 326]

3 J.Tarazona y E. Vilela, «Vulnerabilidad actual y futura al cambio climático en la bahía de Sechura y propuestas de adaptación en Perú», en Vulnerabilidad y adaptación al cambio climático en el Perú: Prioridades de ciencia y tecnología, CONCYTEC, Lima, Perú, 2009, pp. 95 - 114 [p 108] 